\title{
Effects of NAMPT polymorphisms and haplotypes on circulating visfatin/NAMPT levels in hypertensive disorders of pregnancy
}

\author{
Marcelo R Luizon ${ }^{1}$, Vanessa A Belo ${ }^{1}$, Ana CT Palei ${ }^{2}$, Lorena M Amaral ${ }^{1}$, Riccardo Lacchini ${ }^{3}$, \\ Valeria C Sandrim ${ }^{4}$, Geraldo Duarte ${ }^{5}$, Ricardo C Cavalli ${ }^{5}$ and Jose E Tanus-Santos ${ }^{1}$
}

Dysregulation of adipocytokines may be associated with endothelial dysfunction in women with preeclampsia (PE), who are at increased risk of future cardiovascular disease. Visfatin, an adipocytokine with a potential cardiovascular role, is also known as nicotinamide phosphorybosil transferase (NAMPT). NAMPT gene polymorphisms affect circulating visfatin/NAMPT levels in obesity. Most findings provide evidence for increased visfatin/NAMPT circulating levels in PE. However, no previous study has tested the hypothesis that NAMPT polymorphisms affect visfatin/NAMPT levels in hypertensive disorders of pregnancy. We studied the effects of the NAMPT polymorphisms T $>C$ ( $r$ 1319501) and A $>$ G (rs3801266), and the haplotypes formed by them on visfatin/NAMPT levels and whether these genetic markers are associated with gestational hypertension (GH) and PE. We studied 212 healthy pregnant (HP), 181 patients with GH and 208 with PE. Genotypes were determined by Taqman allele discrimination assays. Plasma visfatin/NAMPT levels were measured by ELISA. No significant differences in visfatin/NAMPT levels were found among the groups. However, higher visfatin/NAMPT levels $(P<0.05)$ were found in GH patients carrying the AG or the GG genotypes for the rs3801266 polymorphism or the ' $T$, $G$ ' haplotype. The TC and CC genotypes and the $C$ allele for the rs1319501 polymorphism were more frequent in the HP than in the PE group $(P<0.05)$. Moreover, the ' $C$, A' haplotype was also more frequent in the HP than in the PE group $(P<0.01)$. Our findings suggest that although the rs3801266 polymorphism and the ' $T$, G' haplotype affect visfatin/NAMPT levels in GH, the rs1319501 polymorphism and the 'C, A' haplotype affect the susceptibility to PE.

Hypertension Research (2015) 38, 361-366; doi:10.1038/hr.2015.15; published online 26 February 2015

Keywords: adipocytokine; gestational hypertension; haplotypes; NAMPT; preeclampsia; visfatin

\section{INTRODUCTION}

Hypertensive disorders of pregnancy are associated with increased maternal and perinatal mortality and morbidity, and affect up to $10 \%$ of pregnancies. ${ }^{1}$ Although the mechanisms responsible for preeclampsia (PE) are not fully elucidated, reduced placental perfusion is postulated as an initiating mechanism, which leads to widespread dysfunction of the maternal vascular endothelium and hypertension. ${ }^{2,3}$

Women with a history of $\mathrm{PE}$ are at increased risk of future cardiovascular disease (CVD). ${ }^{4,5}$ Endothelial, vascular and metabolic dysfunction during PE are among the potential mechanisms underlying the increased risk of CVD. ${ }^{4}$ Obesity is a major risk factor for both $\mathrm{PE}$ and $\mathrm{CVD},{ }^{6,7}$ and dysregulated adipocytokine release by adipocytes may be associated with endothelial dysfunction in preeclamptic women. ${ }^{8}$ In addition, adipocytokines may have a role in the critical process of trophoblast invasion and successful placentation, which requires appropriate angiogenesis, as previously reviewed. ${ }^{9}$ Therefore, adipocytokines may be of pathophysiological significance for both $\mathrm{PE}^{10,11}$ and many CVD including hypertension. ${ }^{12}$

Visfatin is a recently described adipocytokine, also known as nicotinamide phosphorybosil transferase (NAMPT), which has a potential role in the pathophysiology of metabolic disorders such as hypertension and obesity. ${ }^{13}$ Visfatin/NAMPT was proposed as a biomarker of endothelial dysfunction and vascular damage, but further studies are required to confirm visfatin/NAMPT as a target in cardiometabolic diseases. ${ }^{14}$ Previous studies reviewed the contribution of visfatin/NAMPT in PE. ${ }^{15}$ Most findings provide evidence for increased circulating visfatin levels ${ }^{16-20}$ in patients with PE, although decreased serum visfatin levels ${ }^{21}$ or visfatin expression have also been

\footnotetext{
${ }^{1}$ Department of Pharmacology, Ribeirao Preto Medical School, University of Sao Paulo, Ribeirao Preto, Brazil; ${ }^{2}$ Department of Pharmacology, University of Mississippi Medical Center, Jackson, MS, USA; ${ }^{3}$ Department of Psychiatric Nursing and Human Sciences, Ribeirao Preto College of Nursing, University of Sao Paulo, Ribeirao Preto, Brazil; ${ }^{4}$ Department of Pharmacology, Institute of Biosciences, Universidade Estadual Paulista (UNESP), Botucatu, Brazil and ${ }^{5}$ Department of Gynecology and Obstetrics, Ribeirao Preto Medical School, University of Sao Paulo, Ribeirao Preto, Brazil

Correspondence: Dr MR Luizon, Department of Pharmacology, Ribeirao Preto Medical School, University of Sao Paulo, Avenue Bandeirantes 3900, 14049-900 Ribeirao Preto, Brazil. 
reported in PE. ${ }^{22}$ Moreover, other studies found no differences in circulating visfatin levels when normal pregnant were compared with patients with PE. ${ }^{23,24}$

We have recently reported that haplotypes of the NAMPT gene affect plasma visfatin/NAMPT levels in healthy but not in obese children and adolescents. ${ }^{25}$ We focused on single-nucleotide polymorphisms (SNPs) in the promoter region ( $\mathrm{T}>\mathrm{C}, \mathrm{rs1319501)}$ and in the intron $1(\mathrm{~A}>\mathrm{G}, \mathrm{rs} 3801266)$ of the NAMPT gene, which have been associated with obesity and CVDs. ${ }^{25-27}$ However, no previous study has examined whether NAMPT polymorphisms and haplotypes affect plasma visfatin/NAMPT levels in hypertensive disorders of pregnancy. It is possible that patients with gestational hypertension $(\mathrm{GH})$ and $\mathrm{PE}$ carrying specific NAMPT genotypes or haplotypes have different plasma visfatin/NAMPT levels.

In the present study, we aimed at comparing the plasma visfatin/ NAMPT levels in healthy pregnant (HP) with those found in patients with GH or PE. We then examined whether NAMPT polymorphisms or haplotypes affect plasma visfatin/NAMPT levels in the HP, GH and $\mathrm{PE}$ groups, and whether they are associated with susceptibility to $\mathrm{GH}$ and PE.

\section{METHODS}

\section{Study population}

Approval for use of human subjects was obtained from the Institutional Review Board at the Ribeirao Preto Medical School, University of Sao Paulo. All volunteers were consecutively enrolled at the Department of Obstetrics and Gynecology, University Hospital. We studied 601 pregnant: 212 HP with uncomplicated pregnancies, 181 women with GH and 208 women with PE. Hypertensive disorders were defined in accordance with the National High Blood Pressure Education Program Working Group on High Blood Pressure in Pregnancy. ${ }^{28} \mathrm{GH}$ was defined as pregnancy-induced hypertension $(\geqslant 140$ $\mathrm{mm} \mathrm{Hg}$ systolic or $\geqslant 90 \mathrm{~mm} \mathrm{Hg}$ diastolic on two or more measurements at least $6 \mathrm{~h}$ apart) in a woman after 20 weeks of gestation, and returning to normal by 12 weeks post-partum. PE was defined as GH plus significant proteinuria $(\geqslant 0.3 \mathrm{~g}$ per $24 \mathrm{~h})$. No women with pre-existing hypertension, with or without superimposed PE, were included in the present study. Methyldopa was the initial antihypertensive treatment during pregnancy. If the pregnant did not respond to methyldopa, nifedipine and/or hydralazine were added to achieve desired blood pressure levels.

At the time of clinic attendance, written informed consent was provided and maternal venous blood samples were collected. Genomic DNA was extracted from the cellular component of $1 \mathrm{ml}$ of whole blood by a salting-out method and stored at $-20{ }^{\circ} \mathrm{C}$ until analyzed. Plasma samples were obtained after centrifugation of whole blood, collected into tubes containing EDTA and centrifuged at $2000 \mathrm{~g}$ for $10 \mathrm{~min}$. Those samples were stored at $-70^{\circ} \mathrm{C}$ until assayed.

\section{Enzyme immunoassays of visfatin/NAMPT}

Visfatin/NAMPT concentrations were measured in EDTA-plasma using a kit (RayBio Human Visfatin EIA-VIS-1, Norcross, GA, USA) according to the manufacturer's instructions. The intra-assay and inter-assay coefficient of variation were $<10.0 \%$, and $<15.0 \%$, respectively. The samples that showed concentrations below the limit of detection of the assay $(0.778 \mathrm{ng} / \mathrm{ml})$ were reanalyzed.

\section{Genotype determination}

Genotypes for two SNPs were determined: the $\mathrm{T}>\mathrm{C}$ at position -423 in the promoter region (dbSNP ID: rs1319501; Assay ID: C_7590641_30), and A > G in the intron 1 (dbSNP ID: rs3801266; Assay ID: C_340124_10) of NAMPT gene were determined by Taqman Allele Discrimination Assays (Applied Biosystems, Carlsbad, CA, USA). Real-time PCR was performed in a total volume of $12 \mu \mathrm{l}$ ( $3 \mathrm{ng}$ of DNA, $1 \times$ TaqMan master mix, $1 \mathrm{x}$ assay mix) placed in 96-well PCR plates. Fluorescence from PCR amplification was detected using StepOne Plus Real-Time PCR device (Applied Biosystems) and analyzed with manufacturer's software.

\section{Statistical analysis}

The clinical characteristics and the effects of genotypes and haplotypes on circulating visfatin/NAMPT levels were compared by analysis of variance followed by Tukey test (normally distributed variables) or Kruskall-Wallis test followed by Dunn's Multiple Comparison test (not normally distributed variables). The categorical variables were compared by $\chi^{2}$ tests. The distribution of genotypes for each polymorphism was assessed for deviation from the Hardy-Weinberg equilibrium, and differences in genotype frequency and in allele frequency were assessed using $\chi^{2}$ tests. A value of $P<0.05$ was considered

Table 1 Clinical and demographic characteristics of the study subjects

\begin{tabular}{|c|c|c|c|c|c|}
\hline Parameters & Healthy pregnant $(n=212)$ & Gestational hypertension $(\mathrm{n}=181)$ & $\mathrm{P}$ values & Preeclampsia $(\mathrm{n}=208)$ & $\mathrm{P}$ values \\
\hline Age (years) & $24.55 \pm 0.41$ & $27.27 \pm 0.51^{a}$ & 0.001 & $26.59 \pm 0.46^{a}$ & 0.001 \\
\hline Ethnicity (\% White) & 68.22 & 72.40 & 0.789 & 71.20 & 0.524 \\
\hline Current smoking (\%) & 9.0 & 11.0 & 0.521 & 8.6 & 0.892 \\
\hline $\mathrm{BMI}\left(\mathrm{kg} \mathrm{m}^{-2}\right)$ & $23.29 \pm 0.35$ & $28.18 \pm 0.69^{a}$ & 0.000 & $27.34 \pm 0.46^{a}$ & 0.000 \\
\hline SBP (mm Hg) & $110.6 \pm 0.79$ & $133.1 \pm 1.34^{a}$ & 0.000 & $140.1 \pm 1.46^{a, b}$ & 0.000 \\
\hline DPB (mm Hg) & $71.54 \pm 0.64$ & $83.72 \pm 0.95^{a}$ & 0.000 & $88 \pm 0.88^{a, b}$ & 0.000 \\
\hline HR (beats per min) & $82.01 \pm 0.64$ & $81.56 \pm 0.56$ & 0.598 & $82.34 \pm 0.63$ & 0.714 \\
\hline Fasting glucose (mg dl-1) & $75.04 \pm 1.04$ & $78.56 \pm 1.04^{a}$ & 0.018 & $80.69 \pm 1.78^{a}$ & 0.006 \\
\hline $\mathrm{Hb}\left(\mathrm{g} \mathrm{dl}^{-1}\right)$ & $11.87 \pm 0.13$ & $11.89 \pm 0.09$ & 0.900 & $11.89 \pm 0.10$ & 0.905 \\
\hline Hct $(\%)$ & $35.65 \pm 0.44$ & $35.77 \pm 0.27$ & 0.808 & $35.92 \pm 0.29$ & 0.598 \\
\hline Creatinine $(\mu \mathrm{mol} \mathrm{I}-1)$ & $66.7 \pm 2.8$ & $62.1 \pm 0.9$ & 0.260 & $70.4 \pm 1.6^{b}$ & 0.632 \\
\hline 24- h Pr (mg per 24 h) & ND & $65.94 \pm 8.1$ & & $874.2 \pm 110.2^{b}$ & 0.000 \\
\hline Primiparity (\%) & 49.2 & 39.8 & 0.067 & 44.2 & 0.318 \\
\hline GAD (weeks) & $39.8 \pm 0.1$ & $38.9 \pm 0.1^{a}$ & 0.000 & $36.1 \pm 0.3^{a}$ & 0.000 \\
\hline Newborn weight (g) & $3297 \pm 34.6$ & $3201.0 \pm 41.7$ & 0.095 & $2542.0 \pm 64.7^{a}$ & 0.000 \\
\hline GAS (weeks) & $36.8 \pm 0.2$ & $36.0 \pm 0.5$ & 0.330 & $34.1 \pm 0.2^{\mathrm{a}}$ & 0.000 \\
\hline Antihypertensive treatment (\%) & ND & 60.7 & & 61 & 0.977 \\
\hline
\end{tabular}

Abbreviations: BMI, body mass index; DBP, diastolic blood pressure; GAD, gestational age at delivery; GAS, gestational age at sampling; Hb, hemoglobin concentration; Hct, hematocrit; HR, heart rate; ND, not determined (however, negative dipstick test); SBP, systolic blood pressure; $24-\mathrm{h} \mathrm{Pr}$, 24-h proteinuria.

Antihypertensive treatment: methyldopa, nifedipine and hydralazine. Values are the mean + s.e.m.

a $P<0.05$ vs. healthy pregnant group.

${ }^{\mathrm{b}} P<0.05$ vs. gestational hypertension group.

Significant $P$ values are in bold. 
significant. Given the sample size of this study, the detectable odds ratio is 1.5 considering a statistical power of $80 \%$ and an alpha of 0.05 , calculated using the PGA Matlab software. ${ }^{29}$

Haplotype frequencies were estimated by using Haplo.stats package version 1.4.4 (http://cran.r-project.org/web/packages/haplo.stats/index.html), as described in detail elsewhere. ${ }^{30,31}$ The possible haplotypes including the

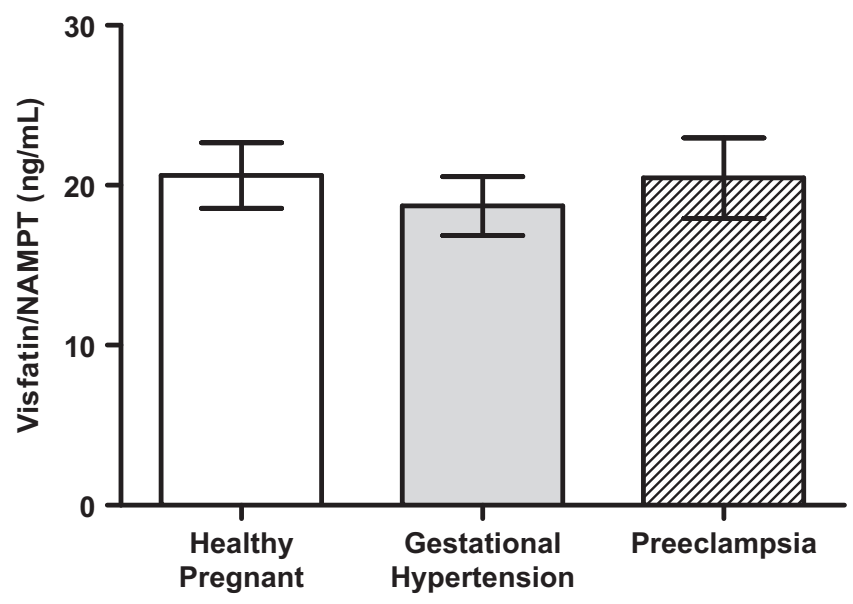

Figure 1 Plasma visfatin/nicotinamide phosphorybosil transferase (NAMPT) concentrations in healthy pregnant $(N=107)$, patients with gestational hypertension $(N=105)$ and preeclampsia $(N=90)$. The bars show the mean \pm s.e.m. alleles of the two NAMPT polymorphisms $\mathrm{T}>\mathrm{C}$ (rs1319501) and $\mathrm{A}>\mathrm{G}$ (rs3801266) were: 'T, A', 'T, G', 'C, A' and 'C, G'. However, we have excluded the ' $\mathrm{C}, \mathrm{G}$ ' haplotype from the analysis because of its low frequency. Differences in haplotype frequencies were tested using $\chi^{2}$ tests, and a value of $P<0.0125$ $(0.05 / 4$, the number of haplotypes) was considered significant to correct for the number of comparisons made (Bonferroni's correction). Linkage disequilibrium (LD) was assessed by calculating $\mathrm{D}^{\prime}$ using Haploview software (version 4.2; http://www.broad.mit.edu/mpg/haploview/). Multivariate logistic regression analysis was performed by using the JMP software 5.0.1a (SAS Institute, Cary, NC, USA). Age, gestational age at delivery, fasting glucose and body mass index were considered as independent variables.

To further examine the effects of NAMPT haplotypes on visfatin/NAMPT levels, we have also performed an additional analysis. We compared NAMPT haplotypes distributions in two groups of HP, GH and PE patients: the lower and the upper groups, which included subjects with the lower and upper values of plasma visfatin/NAMPT levels distribution, respectively.

\section{RESULTS}

The clinical and demographic characteristics of pregnant enrolled in this study are shown in Table 1. HP, GH and PE women showed similar ethnicity (\% white), \% current smoking, hemoglobin, hematocrit and creatinine (all $P>0.05$ ). As expected, PE and GH presented higher systolic and diastolic blood pressure compared with HP (both $P<0.05)$. It should be noted, however, that most patients were receiving antihypertensive therapy. The percentage of $\mathrm{GH}$ and $\mathrm{PE}$ patients who received antihypertensive treatment is shown in Table 1. $\mathrm{GH}$ and PE were older than HP $(P<0.05)$. Increased body mass index and fasting glucose was found in $\mathrm{GH}$ and $\mathrm{PE}$ patients compared with

Table 2 NAMPT genotypes and allele frequencies in healthy pregnant, gestational hypertension and preeclampsia groups

\begin{tabular}{|c|c|c|c|c|c|c|c|}
\hline $\begin{array}{l}\text { Genotype or } \\
\text { allele }\end{array}$ & $\begin{array}{l}\text { Health pregnant } \\
\qquad(\mathrm{n}=212, \%)\end{array}$ & $\begin{array}{l}\text { Gestational hypertension } \\
\qquad(\mathrm{n}=181, \%)\end{array}$ & OR $(95 \% \mathrm{Cl})$ & $\begin{array}{c}\mathrm{P} \\
\text { values }\end{array}$ & $\begin{array}{l}\text { Preeclampsia } \\
(n=208, \%)\end{array}$ & OR $(95 \% \mathrm{Cl})$ & $\begin{array}{c}\mathrm{P} \\
\text { values }\end{array}$ \\
\hline \multicolumn{8}{|c|}{$r s 1319501 T>C$} \\
\hline $\mathrm{TT}$ & $117(55)$ & $108(60)$ & 1.000 (Reference) & - & $141(68)$ & 1.000 (Reference) & - \\
\hline $\mathrm{TC}$ & $77(36)$ & $63(35)$ & $0.886(0.580-1.354)$ & 0.576 & $58(28)$ & $0.625(0.411-0.951)$ & $0.027^{a}$ \\
\hline $\mathrm{CC}$ & $18(9)$ & $10(5)$ & $0.602(0.266-1.361)$ & 0.235 & $9(4)$ & $0.415(0.179-0.958)$ & $0.034^{\mathrm{a}}$ \\
\hline $\mathrm{T}$ & $311(73)$ & $279(77)$ & 1.000 (Reference) & - & $340(82)$ & 1.000 (Reference) & - \\
\hline C & $113(27)$ & $83(23)$ & $0.819(0.591-1.135)$ & 0.229 & $76(18)$ & $0.615(0.443-0.855)$ & $0.003^{a}$ \\
\hline \multicolumn{8}{|c|}{$r s 3801266 A>G$} \\
\hline$A A$ & $108(51)$ & $86(48)$ & 1.00 (Reference) & - & $94(45)$ & 1.000 (reference) & - \\
\hline$A G$ & $84(40)$ & $86(48)$ & $1.286(0.850-1.944)$ & 0.232 & $96(46)$ & $1.06(0.68-1.66)$ & 0.184 \\
\hline GG & $20(9)$ & $9(4)$ & $0.565(0.244-1.304)$ & 0.177 & $18(9)$ & $1.14(0.66-1.97)$ & 0.924 \\
\hline$A$ & $300(71)$ & $258(71)$ & 1.000 (Reference) & & $284(68)$ & 1.000 (Reference) & \\
\hline G & $124(29)$ & $104(29)$ & $0.975(0.716-1.330)$ & 0.874 & $132(32)$ & $1.124(0.838-1.509)$ & 0.434 \\
\hline
\end{tabular}

Abbreviations: $\mathrm{Cl}$, confidence interval; NAMPT, nicotinamide phosphorybosil transferase gene; OR, odds ratio.

a $P<0.05$ vs. health pregnant group.

Table 3 NAMPT haplotypes frequencies in healthy pregnant, gestational hypertension and preeclampsia groups

\begin{tabular}{|c|c|c|c|c|c|c|c|}
\hline Haplotype & $\begin{array}{l}\text { Healthy pregnant } \\
\qquad(R F ; \mathrm{n}=424)\end{array}$ & $\begin{array}{l}\text { Gestational hypertension } \\
\qquad(R F ; \mathrm{n}=362)\end{array}$ & $\begin{array}{c}\mathrm{P} \\
\text { values }\end{array}$ & OR $(95 \% \mathrm{Cl})$ & $\begin{array}{l}\text { Preeclampsia } \\
(R F ; \mathrm{n}=416)\end{array}$ & $\begin{array}{c}\mathrm{P} \\
\text { values }\end{array}$ & OR $(95 \% \mathrm{Cl})$ \\
\hline $\mathrm{T}, \mathrm{A}$ & 0.4454 & 0.4834 & 0.2725 & 1.0000 (Reference) & 0.5146 & 0.0754 & 1.000 (Reference) \\
\hline $\mathrm{T}, \mathrm{G}$ & 0.2880 & 0.2872 & 0.9304 & $0.9222(0.6583-1.2919)$ & 0.3026 & 0.4918 & $0.9288(0.6735-1.2809)$ \\
\hline \multirow[t]{2}{*}{ C, A } & 0.2621 & 0.2292 & 0.2672 & $0.8216(0.5842-1.1555)$ & 0.1680 & $0.0025^{a}$ & $0.5688(0.3962-0.8164)$ \\
\hline & & \multicolumn{3}{|c|}{ Global-stat $=2.18343, \mathrm{df}=3, P=0.53522^{\mathrm{b}}$} & \multicolumn{3}{|c|}{ Global-stat $=10.509, \mathrm{df}=3, P=0.014702^{a, c}$} \\
\hline
\end{tabular}

Abbreviations: $\mathrm{Cl}$, confidence intervals; $\mathrm{NA}$, not available; NAMPT, nicotinamide phosphorybosil transferase gene; OR, odds ratio; RF, relative frequency.

Global Score Statistics:

aStatistically significant: $P<0.05$ vs. healthy pregnant group.

bGestational hypertension vs. healthy pregnant.

cPreeclampsia vs. healthy pregnant. 


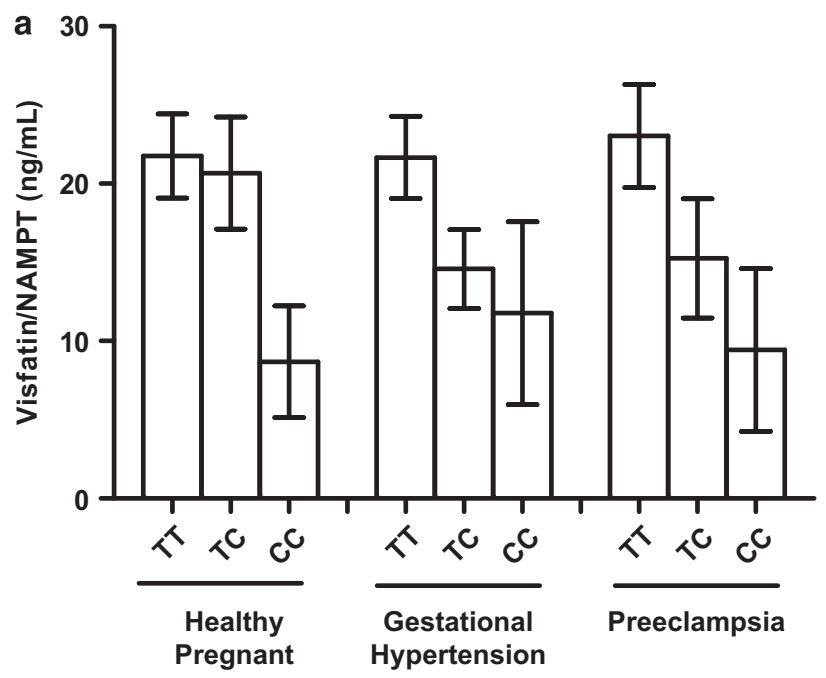

HP group (all $P<0.05$ ). We found lower gestational age at delivery $(\mathrm{GAD})$ in $\mathrm{GH}$ and $\mathrm{PE}$, and lower newborn weights and gestational age at sampling in $\mathrm{PE}($ all $P<0.05)$ compared with HP. Significant proteinuria was found in $\mathrm{PE}$.

For technical reasons, we were not able to measure the plasma visfatin/NAMPT levels for all subjects enrolled in the study. Therefore, the values are shown for $107 \mathrm{HP}$ women, 105 patients with GH and 90 patients with PE (Figure 1). We found no significant differences in visfatin/NAMPT levels among $\mathrm{HP}, \mathrm{GH}$ and $\mathrm{PE}(P>0.05$; Figure 1$)$.

Genotypes and alleles distribution are shown in Table 2. The distribution of genotypes for each polymorphism showed no deviation from Hardy-Weinberg equilibrium. The TC and CC genotypes and the $\mathrm{C}$ allele for the $\mathrm{T}>\mathrm{C}$ (rs1319501) polymorphism were found at higher frequency in HP when compared with PE group $(P<0.05$; Table 2). The associations of the $C$ allele and the CC genotype were confirmed in the multivariate logistic regression analysis adjusted for independent variables (Supplementary Table 1). Conversely, we found no significant differences in genotype and allele frequencies for the $A>G$ (rs3801266) polymorphism when the groups were compared $(P>0.05$; Table 2$)$. Haplotypes distributions are shown in Table 3.
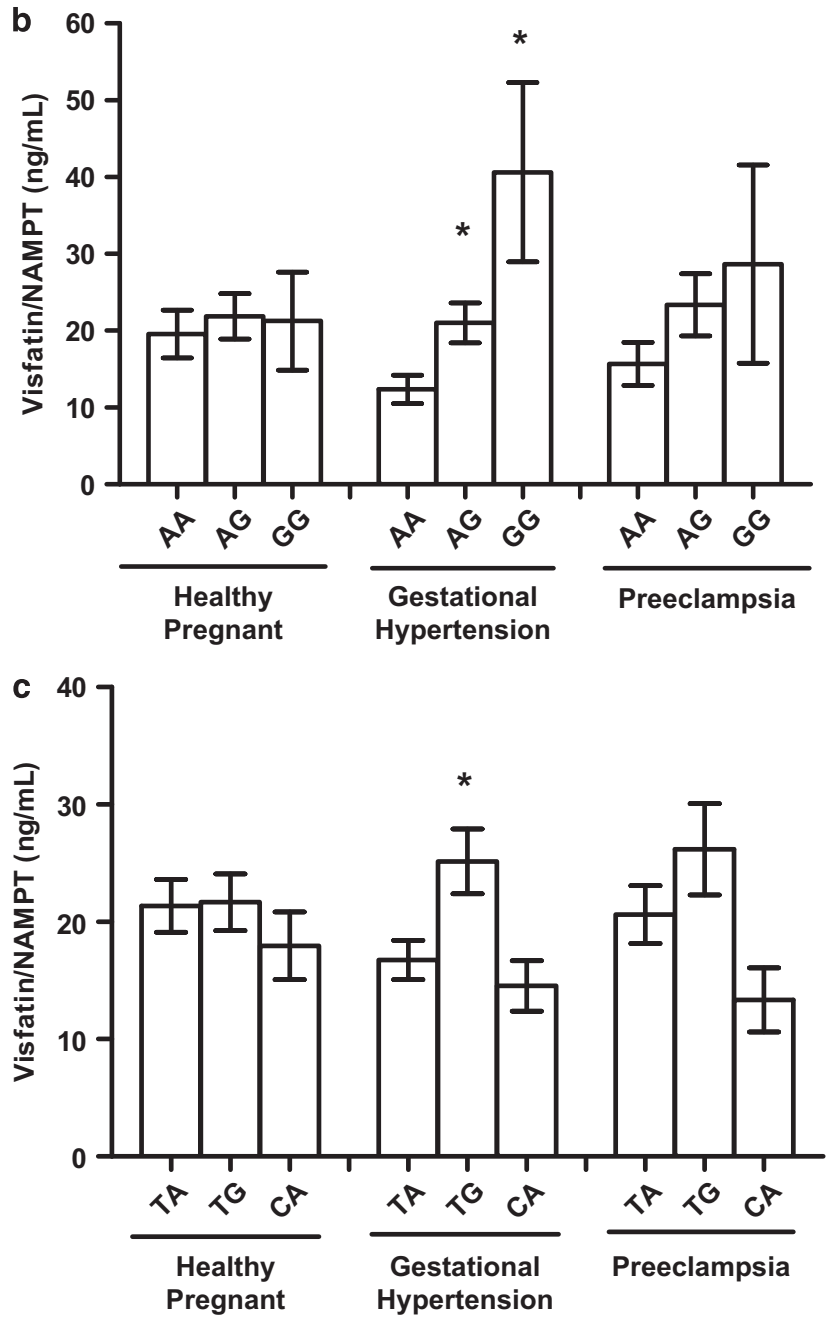

The ' $\mathrm{C}$, A' haplotype was more common in the HP group than in the PE group $(P<0.01$; Table 3$)$. Conversely, we found no significant differences when the HP and the GH groups were compared $(P>0.05$; Table 3).

We examined the effects of NAMPT genotypes and haplotypes on plasma visfatin/NAMPT levels. We found no effects of the different genotypes for the $\mathrm{T}>\mathrm{C}$ (rs1319501) polymorphism on plasma visfatin/NAMPT levels $(P>0.05$; Figure $2 \mathrm{a})$. Conversely, we found that $\mathrm{GH}$ patients carrying the $\mathrm{AG}$ and $\mathrm{GG}$ genotypes for the $\mathrm{A}>\mathrm{G}$ (rs3801266) polymorphism showed higher visfatin/NAMPT levels than GH patients carrying the AA genotype $(P<0.05$; Figure $2 \mathrm{~b})$. We were able to detect the effects of different genotypes for the NAMPT polymorphism rs3801266 on visfatin/NAMPT levels with a power of $56 \%$. Regarding the haplotypes, GH patients carrying the ' $\mathrm{T}$, G' haplotype showed higher plasma visfatin/NAMPT levels than the other haplotypes $(P<0.05$; Figure $2 \mathrm{c})$, but we found no significant differences when comparing different haplotypes within the HP and PE groups $(P>0.05$; Figure 2c).

We performed a further analysis and compared the lower and upper groups of plasma visfatin/NAMPT values (Table 4). We confirmed that the ' $\mathrm{T}, \mathrm{G}$ ' haplotype was more common in the upper group when considering the $\mathrm{GH}$ patients $(P<0.05$; Table 4$)$, but we found no differences between the lower and upper groups when considering HP and $\mathrm{PE}$ patients (Supplementary Tables 2 and 3, respectively). However, it should be noted that the ' $\mathrm{C}, \mathrm{A}$ ' haplotype tended to be more frequent in the lower group of plasma visfatin/NAMPT values when considering either GH $(P=0.056$, Table 4$)$ or PE patients $(P=0.062$, Supplementary Table 3$)$.

\section{DISCUSSION}

The main novel findings reported here were that (1) GH patients carrying the AG and GG genotypes for the NAMPT A > G (rs3801266) polymorphism and the ' $\mathrm{T}, \mathrm{G}$ ' haplotype showed increased plasma visfatin/NAMPT levels, and that (2) the TC and CC genotypes and the C allele for the NAMPT T $>C$ (rs1319501) polymorphism and the ' $\mathrm{C}$, A' haplotype may protect against PE.

Discordant findings have been reported regarding maternal circulating visfatin/NAMPT levels patients with PE. Most studies provide evidence for increased plasma visfatin levels in PE as compared with normotensive pregnant ${ }^{16,20}$ or increased serum visfatin levels in $\mathrm{PE}^{17,19}$ and in the 11-13th weeks of pregnancy in women who 
Table 4 NAMPT haplotypes frequency distribution in the lower and in the upper groups of visfatin/NAMPT levels for the patients with gestational hypertension

\begin{tabular}{|c|c|c|c|c|c|}
\hline Haplotype & Hap-score & Lower $(R F ; \mathrm{n}=105)$ & Upper $(R F ; \mathrm{n}=105)$ & $\mathrm{P}$ values & OR $(95 \% \mathrm{Cl})$ \\
\hline $\mathrm{T}, \mathrm{A}$ & -0.2666 & 0.4615 & 0.4434 & 0.7897 & 1.0000 (Reference) \\
\hline $\mathrm{T}, \mathrm{G}$ & 2.1672 & 0.2596 & 0.3867 & $0.0302^{\mathrm{a}}$ & $1.8148(0.9007-3.6566)$ \\
\hline C, A & -1.9090 & 0.2788 & 0.1698 & 0.0562 & $0.6152(0.2991-1.2653)$ \\
\hline
\end{tabular}

Abbreviations: $\mathrm{Cl}$, confidence interval; $\mathrm{NA}$, not available; NAMPT, nicotinamide phosphorybosil transferase; OR, odds ratio; RF, relative frequencies.

Global Score Statistics: global-stat $=6.39231, \mathrm{df}=2, P=0.04092^{\mathrm{a}}$.

aStatistically significant: $P<0.05$ vs. Lower group.

developed PE. ${ }^{18}$ Conversely, other studies showed decreased serum visfatin levels in $\mathrm{PE}^{21}$ or decreased visfatin expression in the placental bed in PE as compared with normotensive controls. ${ }^{22}$ However, we found no significant differences in plasma visfatin/NAMPT levels when we compared the HP, GH and PE groups. Our findings are supported by other studies showing no differences in maternal plasma or serum visfatin concentrations in normal pregnant compared with patients with $\mathrm{PE}^{23}$ or to patients with $\mathrm{GH} .{ }^{24}$ Although we have no precise explanation for these discordant findings, they may be attributed to differences between the studied populations, differences between plasma and serum, differences in sensitivity and specificity of different visfatin immunoassays, ${ }^{32}$ and to differences of gestational age at sampling.

Although no study has examined the effects of NAMPT polymorphisms on circulating visfatin/NAMPT levels in hypertensive disorders of pregnancy, we have previously found increased plasma visfatin/NAMPT levels in control and obese subjects carrying the GG genotype for the NAMPT A $>\mathrm{G}$ (rs3801266) polymorphism. ${ }^{25}$ Moreover, the ' $\mathrm{T}, \mathrm{G}$ ' haplotype was associated with higher plasma visfatin/ NAMPT levels in control subjects, but not in obese children and adolescents. ${ }^{25}$ These results are in line with our findings showing that the AG and the GG genotypes for the A>G (rs3801266) polymorphism and the ' $\mathrm{T}, \mathrm{G}$ ' haplotype are associated with higher plasma visfatin/NAMPT levels in GH patients, but not in PE patients. Together, these findings indicate that NAMPT polymorphisms and haplotypes have important effects on plasma visfatin/NAMPT levels, but these effects may vary under different physiological and/or disease conditions. Although no functional study was carried out to show how the $A>G$ (rs3801266) polymorphism affects visfatin/NAMPT expression, other factors may also affect visfatin/NAMPT expression independent of their genotypes. However, our results suggest that the rs3801266 polymorphism is relevant and affects visfatin/NAMPT levels in $\mathrm{GH}$ patients.

To our knowledge, no previous study has examined whether NAMPT polymorphisms or haplotypes are associated with hypertensive disorders of pregnancy. We showed for the first time that the TC and CC genotypes and the $\mathrm{C}$ allele for the $\mathrm{T}>\mathrm{C}$ (rs1319501) polymorphism, as well as the ' $\mathrm{C}, \mathrm{A}$ ' haplotype may protect against PE. Consistent with this finding, the ' $\mathrm{C}$, $\mathrm{A}$ ' haplotype tended to be more frequent in the lower group when compared the upper and lower groups of plasma visfatin/NAMPT levels for both GH (Table 4) and PE (Supplementary Table 3) patients. Together, our findings suggest that the ' $\mathrm{C}, \mathrm{A}$ ' haplotype has a protective effect against PE because it may be associated with lower plasma visfatin/NAMPT levels. PE shares metabolic risk factors with CVD, including obesity, ${ }^{6,7}$ and visfatin/NAMPT levels are enhanced under those pathological conditions. ${ }^{15,25}$ Moreover, visfatin/NAMPT was proposed as a biomarker of endothelial dysfunction, ${ }^{14}$ a potential mechanism underlying the increased cardiovascular risk in women with history of PE. ${ }^{4,5}$
However, here we have not studied the relationship between visfatin/NAMPT levels or other relevant factors such as oxidative stress $^{33,34}$ with endothelial dysfunction in PE, and this hypothesis remains to be proved.

In contrast with the $\mathrm{A}>\mathrm{G}$ ( $\mathrm{rs} 3801266$ ) polymorphism, the $\mathrm{T}>\mathrm{C}$ (rs1319501) polymorphism in the promoter region of the NAMPT gene had no effects on plasma visfatin/NAMPT levels in the present study. However, it is likely to affect transcription factor binding according to its Score $2 \mathrm{c}$ at RegulomeDB, ${ }^{35}$ and to the Encyclopedia of DNA Elements (ENCODE) data related to gene regulation around its location in the promoter region of the NAMPT gene (Supplementary Figure 1). Indeed, the $5^{\prime}$-upstream NAMPT region has several regulatory elements, ${ }^{36}$ and a recent in silico analysis of the $5^{\prime}$ NAMPT promoter region revealed putative cis regulatory elements, including the binding sites for the transcription factors NF- $\kappa \beta$, SP1 and STAT. ${ }^{37}$ Interestingly, the location of the $\mathrm{T}>\mathrm{C}$ ( $\mathrm{rs} 1319501)$ polymorphism overlaps not only with these but also with a large number of Transcription Factor ChIP-seq data from ENCODE (Supplementary Figure 2). Together, these findings suggest that the rs1319501 polymorphism may affect NAMPT gene expression.

The T $>$ C polymorphism (rs1319501) could also be in LD with another functional polymorphism in the promoter region of the NAMPT gene. Indeed, an extensive LD structure was reported at the NAMPT locus, ${ }^{38}$ and the promoter polymorphisms rs1319501 and rs9770242 were in complete LD in different populations. ${ }^{39,40}$ In addition, the $\mathrm{LD}$ value between the studied polymorphisms rs1319501 and rs3801266 was $D^{\prime}=0.581$ for the population of European ancestry (CEU) of the 1000 Genomes Project (Supplementary Figure 1). However, we did not examine other NAMPT polymorphisms, especially in the promoter region, which may affect visfatin/NAMPT levels. Importantly, our findings must be replicated in further studies. These findings are relevant because the associated genetic markers may allow early detection of patients at increased risk of hypertensive disorders of pregnancy, which increase morbidity and mortality. ${ }^{41}$

The present study has other limitations. First, we studied a relatively small number of patients and plasma visfatin/NAMPT levels were determined in a smaller number of patients. Despite this limitation, we found significant associations for NAMPT polymorphisms and haplotypes, and it is important to consider that the ' $\mathrm{T}, \mathrm{G}$ ' and ' $\mathrm{C}, \mathrm{A}$ ' haplotypes are common haplotypes ( $>15 \%$ frequency), thus increasing their importance. Second, we have not measured placental or tissue levels of visfatin/NAMPT, and therefore we do not know how marked the effects are at the tissue level.

In conclusion, we found that GH patients carrying the AG and GG genotypes for the NAMPT A $>\mathrm{G}$ (rs3801266) polymorphism and the ' $T$, G' haplotype showed higher plasma visfatin/NAMPT levels, and that the TC and CC genotypes and the $\mathrm{C}$ allele for the NAMPT T $>\mathrm{C}$ (rs1319501) polymorphism, as well as the ' $\mathrm{C}$, A' haplotype may 
protect against PE. We present, for the first time, evidence that NAMPT genotypes and haplotypes affect circulating visfatin/NAMPT levels in $\mathrm{GH}$, and affect the susceptibility to PE. Our findings suggest that NAMPT polymorphisms may be useful to identify a group of women at increased risk of hypertensive disorders of pregnancy.

\section{CONFLICT OF INTEREST}

The authors declare no conflict of interest.

\section{ACKNOWLEDGEMENTS}

This study was supported by the Conselho Nacional de Desenvolvimento Científico e Tecnológico (CNPq-Brazil), the Coordenação de Aperfeiçoamento de Pessoal de Nível Superior (CAPES-Brazil) and the Fundação de Amparo a Pesquisa do Estado de São Paulo (FAPESP-Brazil).

1 Hutcheon JA, Lisonkova S, Joseph KS. Epidemiology of pre-eclampsia and the other hypertensive disorders of pregnancy. Best Pract Res Clin Obstet Gynaecol 2011; 25: 391-403.

2 Palei AC, Spradley FT, Warrington JP, George EM, Granger JP. Pathophysiology of hypertension in pre-eclampsia: a lesson in integrative physiology. Acta Physiol (Oxf) 2013; 208: 224-233.

3 Warrington JP, George EM, Palei AC, Spradley FT, Granger JP. Recent advances in the understanding of the pathophysiology of preeclampsia. Hypertension 2013; 62 666-673.

4 Ahmed R, Dunford J, Mehran R, Robson S, Kunadian V Pre-eclampsia and future cardiovascular risk among women: a review. J Am Coll Cardiol 2014; 63: 1815-1822.

5 Carty DM, Delles C, Dominiczak AF. Preeclampsia and future maternal health. J Hypertens 2010; 28: 1349-1355.

6 Jeyabalan A. Epidemiology of preeclampsia: impact of obesity. Nutr Rev 2013; 71 S18-S25.

7 Roberts JM, Bodnar LM, Patrick TE, Powers RW. The role of obesity in preeclampsia Pregnancy Hypertens 2011; 1: 6-16.

8 Mori T, Shinohara K, Wakatsuki A, Watanabe K, Fujimaki A. Adipocytokines and endothelial function in preeclamptic women. Hypertens Res 2010; 33: 250-254.

9 Tersigni C, Di Nicuolo F, D'Ippolito S, Veglia M, Castellucci M, Di Simone N. Adipokines: new emerging roles in fertility and reproduction. Obstet Gynecol Surv 2011; 66: 47-63.

10 Briana DD, Malamitsi-Puchner A. Reviews: adipocytokines in normal and complicated pregnancies. Reprod Sci 2009; 16: 921-937.

11 Miehle K, Stepan H, Fasshauer M. Leptin, adiponectin and other adipokines in gestational diabetes mellitus and pre-eclampsia. Clin Endocrinol (Oxf) 2012; 76 2-11.

12 Van de Voorde J, Pauwels B, Boydens C, Decaluwe K. Adipocytokines in relation to cardiovascular disease. Metabolism 2013; 62: 1513-1521.

13 Dahl TB, Holm S, Aukrust P, Halvorsen B. Visfatin/NAMPT: a multifaceted molecule with diverse roles in physiology and pathophysiology. Annu Rev Nutr 2012; 32 229-243.

14 Peiro C, Romacho T, Carraro R, Sanchez-Ferrer CF. Visfatin/PBEF/Nampt: a new cardiovascular target? Front Pharmacol 2010; 1: 135

15 Romacho T, Sanchez-Ferrer CF, Peiro C. Visfatin/Nampt: an adipokine with cardiovascular impact. Mediators Inflamm 2013; 2013: 946427.

16 Adali E, Yildizhan R, Kolusari A, Kurdoglu M, Bugdayci G, Sahin HG, Kamaci M. Increased visfatin and leptin in pregnancies complicated by pre-eclampsia. J Matern Fetal Neonatal Med 2009; 22: 873-879.

17 Fasshauer M, Waldeyer T, Seeger J, Schrey S, Ebert T, Kratzsch J, Lossner U, Bluher M, Stumvoll M, Faber R, Stepan H. Serum levels of the adipokine visfatin are increased in pre-eclampsia. Clin Endocrinol (Oxf) 2008; 69: 69-73.

18 Ferreira AF, Rezende JC, de Cassia COR, Akolekar R, Nicolaides KH. Maternal serum visfatin at 11-13 weeks' gestation in preeclampsia. J Hum Hypertens 2013; 27: 261-264.

19 Zorba E, Vavilis D, Venetis CA, Zournatzi V, Kellartzis D, Tarlatzis BC. Visfatin serum levels are increased in women with preeclampsia: a case-control study. J Matern Fetal Neonatal Med 2012; 25: 1668-1673.
20 Zulfikaroglu E, Isman F, Payasli A, Kilic S, Kucur M, Danisman N. Plasma visfatin levels in preeclamptic and normal pregnancies. Arch Gynecol Obstet 2010; 281: 995-998.

$21 \mathrm{Hu}$ W, Wang Z, Wang H, Huang H, Dong M. Serum visfatin levels in late pregnancy and pre-eclampsia. Acta Obstet Gynecol Scand 2008; 87: 413-418.

22 Kim SC, Park MJ, Joo BS, Joo JK, Suh DS, Lee KS. Decreased expressions of vascular endothelial growth factor and visfatin in the placental bed of pregnancies complicated by preeclampsia. J Obstet Gynaecol Res 2012; 38: 665-673.

23 Mazaki-Tovi S, Romero R, Kim SK, Vaisbuch E, Kusanovic JP, Erez O, Chaiworapongsa T, Gotsch F, Mittal P, Nhan-Chang CL, Than NG, Gomez R, Nien JK, Edwin SS, Pacora P, Yeo L, Hassan SS. Could alterations in maternal plasma visfatin concentration participate in the phenotype definition of preeclampsia and SGA? J Matern Fetal Neonatal Med 2010; 23: 857-868.

24 Romao M, Weel IC, Lifshitz SJ, Peracoli MT. Elevated hyaluronan and extracellular matrix metalloproteinase inducer levels in women with preeclampsia. Arch Gynecol Obstet 2014; 289: 575-579.

25 Belo VA, Luizon MR, Lacchini R, Miranda JA, Lanna CM, Souza-Costa DC, Tanus-Santos JE. The effects of NAMPT haplotypes and metabolic risk factors on circulating visfatin/NAMPT levels in childhood obesity. Int J Obes (Lond) 2015; 39 $130-135$.

26 Leander K, Gigante B, Silveira A, Vikstrom M, Hamsten A, Hogberg J. NAMPT (visfatin) and AKT1 genetic variants associate with myocardial infarction. Clin Chim Acta 2012, 413: 727-732

27 Tabassum R, Mahendran Y, Dwivedi OP, Chauhan G, Ghosh S, Marwaha RK, Tandon N Bharadwaj D. Common variants of IL6, LEPR, and PBEF1 are associated with obesity in Indian children. Diabetes 2012; 61: 626-631.

28 Report of the National High Blood Pressure Education Program Working Group on High Blood Pressure in Pregnancy. Am J Obstet Gynecol 2000; 183: S1-S22.

29 Menashe I, Rosenberg PS, Chen BE. PGA: power calculator for case-control genetic association analyses. BMC Genet 2008; 9: 36.

30 Lacchini R, Jacob-Ferreira AL, Luizon MR, Gasparini S, Ferreira-Sae MC, Schreiber R, Nadruz W Jr, Tanus-Santos JE. Common matrix metalloproteinase 2 gene haplotypes may modulate left ventricular remodelling in hypertensive patients. J Hum Hypertens 2012; 26: 171-177

31 Schaid DJ, Rowland CM, Tines DE, Jacobson RM, Poland GA. Score tests for association between traits and haplotypes when linkage phase is ambiguous. $\mathrm{Am} \mathrm{J}$ Hum Genet 2002; 70: 425-434.

32 Korner A, Garten A, Bluher M, Tauscher R, Kratzsch J, Kiess W. Molecular characteristics of serum visfatin and differential detection by immunoassays. J Clin Endocrinol Metab 2007; 92: 4783-4791.

33 Mori T, Watanabe K, Iwasaki A, Kimura C, Matsushita H, Shinohara K, Wakatsuki A. Differences in vascular reactivity between pregnant women with chronic hypertension and preeclampsia. Hypertens Res 2014; 37: 145-150.

34 Pimentel AM, Pereira NR, Costa CA, Mann GE, Cordeiro VS, de Moura RS, Brunini TM Mendes-Ribeiro AC, Resende AC. L-arginine-nitric oxide pathway and oxidative stress in plasma and platelets of patients with pre-eclampsia. Hypertens Res 2013; 36: 783-788.

35 Boyle AP, Hong EL, Hariharan M, Cheng Y, Schaub MA, Kasowski M, Karczewski KJ, Park J, Hitz BC, Weng S, Cherry JM, Snyder M. Annotation of functional variation in personal genomes using RegulomeDB. Genome Res 2012; 22: 1790-1797.

36 Ognjanovic S, Bao S, Yamamoto SY, Garibay-Tupas J, Samal B, Bryant-Greenwood GD. Genomic organization of the gene coding for human pre-B-cell colony enhancing facto and expression in human fetal membranes. J Mol Endocrinol 2001; 26: 107-117.

37 Adyshev DM, Elangovan VR, Moldobaeva N, Mapes B, Sun X, Garcia JG. Mechanical stress induces pre-B-cell colony-enhancing factor/NAMPT expression via epigenetic regulation by miR-374a and miR-568 in human lung endothelium. Am J Respir Cell Mol Biol 2014; 50: 409-418.

38 Zhang YY, Gottardo L, Thompson R, Powers C, Nolan D, Duffy J, Marescotti MC Avogaro A, Doria A. A visfatin promoter polymorphism is associated with low-grade inflammation and type 2 diabetes. Obesity (Silver Spring) 2006; 14: 2119-2126.

39 Bailey SD, Loredo-Osti JC, Lepage P, Faith J, Fontaine J, Desbiens KM, Hudson TJ, Bouchard C, Gaudet D, Pérusse L, Vohl MC, Engert JC. Common polymorphisms in the promoter of the visfatin gene (PBEF1) influence plasma insulin levels in a FrenchCanadian population. Diabetes 2006; 55: 2896-2902.

40 Bottcher Y, Teupser D, Enigk B, Berndt J, Klöting N, Schön MR, Thiery J, Blüher M, Stumvoll M, Kovacs $P$. Genetic variation in the visfatin gene (PBEF1) and its relation to glucose metabolism and fat-depot-specific messenger ribonucleic acid expression in humans. J Clin Endocrinol Metab 2006; 91: 2725-2731.

41 Luizon MR, Sandrim VC, Palei AC, Lacchini R, Cavalli RC, Duarte G, Tanus-Santos JE. Epistasis among eNOS, MMP-9 and VEGF maternal genotypes in hypertensive disorders of pregnancy. Hypertens Res 2012; 35: 917-921.

Supplementary Information accompanies the paper on Hypertension Research website (http://www.nature.com/hr) 